\title{
A Case of the Removal of a Foreign Body From the Hard Palate With the Aid of Nebulized Lidocaine
}

\author{
Kevin M Boehm
}

\begin{abstract}
Although foreign bodies are common chief complaints in pediatrics, foreign bodies in the hard palate are rarely the cause of the patient's complaint. We present the case of a three-year-old with a foreign body retained in the hard palate that we removed with the aid of nebulized lidocaine.
\end{abstract}

Keywords: Pediatric; Foreign body; Palate; Lidocaine

\section{Introduction}

Foreign bodies are a common occurrence in pediatrics, which physicians usually encounter in cases where children accidentally swallow a foreign body or stick objects in their ears or nose. A child with a foreign body stuck to the hard palate, however, is a rare occurrence. We present such a case and our novel approach to treatment, in which we anesthetized the hard palate for foreign body removal.

\section{Case Report}

We present the case of a three-year-old child who presented

Manuscript accepted for publication January 13, 2012

Department of Emergency Medicine, Henry Ford Wyandotte Hospital, Wyandotte MI 48192, USA. Email: kboehm1@hfhs.org

doi:10.4021/jmc499w to the pediatric emergency department with a dime embedded in his hard palate. According to his mother, the child stuck the dime in his mouth and began sucking on it. He then began to cry because he could not get the dime out of his mouth. The dime was lying parallel to his hard palate and the child had sucked the air out between his hard palate and his dime, thereby creating a vacuum effect. When the patient's mother could not remove the dime, she brought the child to the pediatric emergency department. He had stable vital signs and was afebrile with an axillary temperature of $36.5^{\circ} \mathrm{C}\left(97.7^{\circ} \mathrm{F}\right)$, BP $107 / 74$, heart rate of 101 , respirations of $24 /$ minute, and with oxygen saturations of $100 \%$ on room air. He was well developed and 19.1 kilograms. The child was in mild pain distress, but in no respiratory distress. His head, ear, eye, nose, and throat examination revealed the following: a normal cephalic, atraumatic head; bilateral tympanic membranes intact with a good light reflex; eyes with bilateral, equal and reactive pupils with extraoccular muscles intact; a normal nasal exam; an oral exam showing a dime stuck in the hard palate, but otherwise clear and a neck that was supple and without stridor. He was in no respiratory distress and his respiratory exam revealed even, nonlabored breath sounds that were clear bilateral. His heart exam was also typical, revealing a normal S1 and S2 without any accessory sounds and a regular rate. The rest of his physical exam was appropriate for his age.

Upon trying to dislodge the dime with a tongue depressor, the child jumped secondary to pain. We surmised that if we could wedge something between the dime and the hard palate, the vacuum would be released, and the dime would be free. After obtaining informed consent from the mother, we had the child used a standard nebulizer with $4 \mathrm{~mL}$ of $2 \%$ nebulized lidocaine without epinephrine in an attempt to anesthetized the hard palate without needing to use needles or conscious sedation. After the patient finished his nebulized treatment and his oral pharynx was sufficiency anesthetized, he was laid into supine position, under the comfort of mother. We used a tongue blade to keep his mouth open and protect the oral pharynx. At this point, a sterile forceps was used to wedge between the dime and the hard palate, and once suction was released, the dime was freed from the hard palate. The dime left an impression in the hard palate 
with a small abrasion at distal edge. The patient tolerated procedure well. According to the mother, the patient's tetanus status was up to date, and he was discharged home without sequella.

\section{Discussion}

We present this case for two reasons. The first is the rare nature of the hard palate foreign body. A search of the literature reveals that there have been very few reported cases. As of 2008, there have only been 27 reported cases over the last 41 years [1]. The majority of these cases have not been as straightforward as our case. They usually present with concern of neoplasm. Most cases go unnoticed by family until the foreign body has irritated the mucosa of the hard palate, causing an inflammatory response and an overgrowth of the mucosa. When the fleshy nodule is noticed, the case is referred to pediatric otorhinolaryngology for evaluation. Diagnoses from these referrals have included tumor of the hard palate, melanoma of the hard palate, and feeding difficulty. The foreign bodies that have been commonly found include nut shells and screw covers. These are hypothesized to be common hard palate foreign bodies because of the potential to have suction around the periphery as well as the anatomic difference of a child's palate in combination with the nature feeding mechanism $[1,2]$. The dime in our case shares these characteristics, but since our patient presented the day of the event, not enough time passed for the tissue overgrowth that accompanied the other reported cases to take place.

The second reason we presented this case was for our choice of anesthetic. We surmised that if we could get a pair of forceps between the palate and the coin, we could easily remove the coin. The child, however, jumped whenever approached. Based on the author's experience of successfully using nebulized lidocaine for other procedures such as fiberoptic examination of epiglottis, fiberoptic-assisted nasal- tracheal intubation, and awake oral-tracheal intubation, we decided that we would anesthetize the hard palate enough to perform the procedure. We made the correct decision in this case. A review of the literature, however, shows that the use of nebulized lidocaine is an area that needs more research. Currently, there is no reported evidence of the use of nebulized lidocaine in the pediatric population as an anesthetic agent. The only article found was a case report supporting its use for psychogenic cough [3]. Studies on the use of nebulized lidocaine exist in the adult population, but they are limited and contradictory. The limited research examines the use of nebulized lidocaine for the management of cough and asthma symptoms and as an anesthesia for bronchoscopy [4].

\section{Conclusion}

In conclusion, we presented the case of a three-year-old with a retained foreign body in the hard palate that was successfully removed in the pediatric emergency department after the use of nebulized lidocaine. The use of nebulized lidocaine as an anesthetic agent in the pediatric population is nonexistent and is an area that should be explored.

\section{References}

1. Hussain K, Brown AJ, Chavda D. Case report: foreign body in the palate of an infant. Br Dent J. 2008;205(1):2325.

2. de Jong AL, Moola F, Kramer D, Forte V. Foreign bodies of the hard palate. Int J Pediatr Otorhinolaryngol. 1998;43(1):27-31.

3. Sherman JM. Breaking the cycle: lidocaine therapy for habit cough. J Fla Med Assoc. 1997;84(5):308-309.

4. Shirk MB, Donahue KR, Shirvani J. Unlabled use of nebulized medications. Am J Health-Syst Pharm. 2006; 63(18): 1704-1716 